\title{
Short-term potentiation of off-responses in turtle horizontal cells
}

\author{
Abram Akopian ${ }^{1}$, John McReynolds ${ }^{2}$ and Reto Weiler ${ }^{1}$ \\ ${ }^{1}$ Department of Neurobiology, University of Oldenburg, Oldenburg (F.R.G.) and ${ }^{2}$ Department of Physiology, The University of Michigan, \\ Ann Arbor, MI (U.S.A.)
}

(Accepted 30 October 1990)

Key words: Retina; Horizontal cell; Off-response; Light adaptation; Potentiation; Synaptic transmission; Turtle

\begin{abstract}
Depolarizing responses to light off were studied in turtle horizontal cells using intracellular recording in the everted eyecup preparation. In many cells the off-response showed two components (fast and slow) which could overshoot beyond the steady-state dark level. The peak amplitudes of the fast and slow components increased with increasing duration of the light stimulus. A similar enhancement of the off-responses could also be produced by repetitive stimulation with brief flashes. However, the degree of enhancement produced by repetitive stimulation was greater than could be produced by increasing stimulus duration, and the latency of the onset of depolarization was longer, suggesting that the enhancement produced by repetitive stimulation involves an additional mechanism. Dramatic enhancement of the off-response by stimuli which did not affect the on-response during light indicates that the off-response may contain information not present in the on-response. The fast component of the off-response was suppressed to a greater degree than other components by reducing extracellular calcium or in the presence of $500 \mu \mathrm{M}$ cobalt, suggesting that this component may involve a calcium current.
\end{abstract}

\section{INTRODUCTION}

Horizontal cells are second-order neurons in the vertebrate retina which receive excitatory (sign-preserving) synaptic input from photoreceptors, most likely mediated by the neurotransmitter glutamate or a closely related substance ${ }^{1,2,10}$. Like photoreceptors, they respond to light with a graded hyperpolarization. Although the responses of horizontal cells to light have been extensively studied in several species, most of these studies have concentrated on the hyperpolarizing response elicited during light stimulation, a response which is produced by the reduction of transmitter release from photoreceptor terminals. The actual postsynaptic response of the horizontal cells to photoreceptor transmitter, however, is the depolarization that is generated in darkness. The initial part of this EPSP, generally referred to as the off-response, may have several components and contains additional information that is not present in the hyperpolarizing response to light itself.

In turtle horizontal cells it has been shown that the kinetics of the off-response following relatively long ( 2 min) light stimuli became faster with increasing light intensity ${ }^{12}$. The present study shows that 2 major components of the off-response in turtle horizontal cells are also enhanced when the duration of a light stimulus is increased. This enhancement reached a maximum with stimulus durations of a few seconds. An even greater enhancement of these off-response components could be produced by repetitive stimulation with a brief flash. The enhancement of the off-responses was independent of any changes in membrane potential in darkness or during the hyperpolarizing response to light, and may involve modulation of synaptic transmission between photoreceptors and horizontal cells.

\section{MATERIALS AND METHODS}

Responses were recorded from horizontal cells in the inverted eyecup preparation of the red-eared turtle, Pseudemys scripta elegans. Animals were kept in a greenhouse under natural light conditions, and all experiments were performed in the afternoon. The preparation was made under dim room illumination. After decapitating the animal and removing the eye, the posterior portion of the eye was sliced away with a razor blade; after removing as much as possible of the vitreous humor with a paper wick, the eyecup was inverted and mounted with the convex, vitreous side up in the recording chamber, where it was superfused with oxygenated turtle Ringer solution. The composition of the normal Ringer was (in $\mathrm{mM} /$ ) $\mathrm{NaCl}, 110 ; \mathrm{KCl}, 2.6 ; \mathrm{CaCl}_{2}, 2.0 ; \mathrm{MgCl}_{2}, 2.0$; glucose, 10 ; Hepes buffer, 5; adjusted to pH 7.5 with $\mathrm{NaOH}$; known concentrations of test substances were added without substitution. Intracellular recordings were made with conventional electronics and $\mathrm{K}^{+}$ acetate-filled microelectrodes (100-200 M $\Omega$ ). Responses were stored on magnetic tape and displaced on a chartrecorder or digital plotter.

Light stimuli were obtained from a 100-W tungsten-halogen source. In all figures the stimuli were full-field illumination with white light whose intensity was adjusted to produce just saturating 
responses in horizontal cells. The results were obtained from 92 luminosity-type horizontal cell axon terminals, which were identified by their large receptive fields and large hyperpolarizing responses to bright light of all wavelengths. The dark resting potentials of the cells were between -20 and $-35 \mathrm{mV}$. The retina was maintained in a light-adapted state by frequent stimulation with relatively bright, full-field illumination. The results with low calcium were repeated in 4 retinas, and those with the addition of cobalt in 3 retinas. All other experiments were repeated with similar results in at least 10 retinas each.

\section{RESULTS}

At the end of a light stimulus the time-course of the return of the horizontal cell membrane potential to the steady-state dark level often showed several different components, not all of which were seen in every cell. In a few cells the membrane potential returned monotonically to the previous dark level regardless of the type of light stimulus, but in most (about $80 \%$ ) of the cells the off-response following bright stimuli contained two prominent components which are indicated in Fig. 1 as the fast

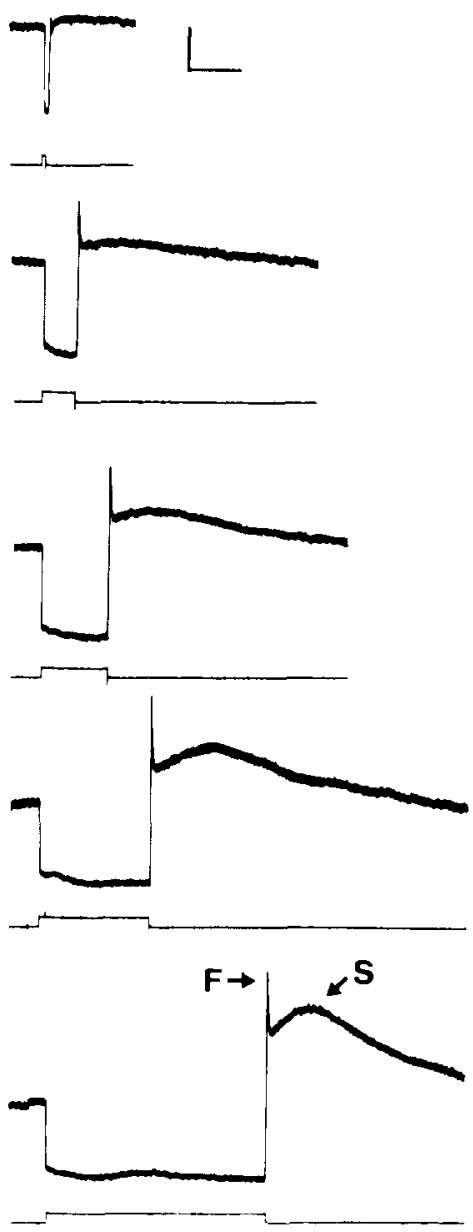

Fig. 1. Off-responses to light stimuli of different durations $(0.3,3$, 6,10 and $20 \mathrm{~s}$ duration). Upward deflection of stimulus monitor (lower trace in each pair) indicates light on. $F$ and $S$ indicate fast and slow components, respectively. Calibration $10 \mathrm{mV}, 5 \mathrm{~s}$.

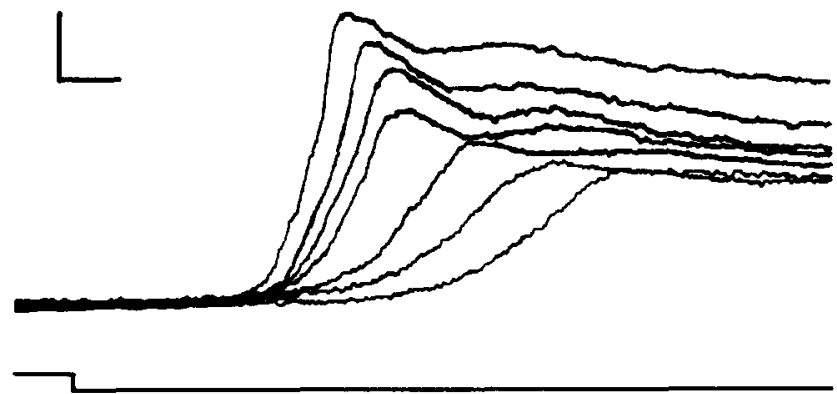

Fig. 2. Effect of stimulus duration on rate of rise and latency of off-response. Superimposed off-responses following stimuli of 0.3 , $0.6,1,2,6,10$ and $15 \mathrm{~s}$ duration. The smallest response is to the $0.3-\mathrm{s}$ stimulus and the largest response is to the 15 -s stimulus. Responses are from different cell than in Fig. 1. Calibration $10 \mathrm{mV}$, $50 \mathrm{~ms}$.

(F) and slow (S) components. The fast component varied considerably, from a small notch on the rising phase of the off-response to a large transient which considerably overshot the steady-state dark potential. The slow component was a relatively long-lasting depolarizing overshoot which gradually declined to the steady-state dark level. In some cells, not used in these experiments, the waveform of the off-response was more complicated, with numerous smaller wavelets and sometimes with pronounced oscillations.

\section{Effect of duration of illumination on the off-response}

Figure 1 shows the off-responses generated by a flash of fixed intensity when the duration of illumination was changed in steps from 0.3 to $20 \mathrm{~s}$. The stimuli were separated by periods of darkness which were long enough to allow the membrane potential and state of adaptation to return to the original condition before the next light stimulus. With increasing duration of the light stimulus the amplitude of both the fast and slow components of the off-response were increased in amplitude. The time required for the development of maximum enhancement of the off-response varied among cells and also varied with light intensity; in the cell shown in Fig. 1, the maximum enhancement of both the fast and slow

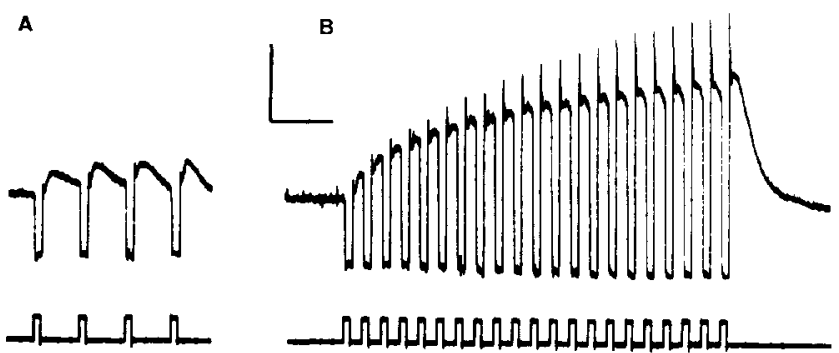

Fig. 3. Off-responses to repetitive stimulation. Flashes $(0.5 \mathrm{~s}$ duration) were separated by dark intervals of $3.5 \mathrm{~s} \mathrm{(A)}$ or $1 \mathrm{~s} \mathrm{(B).}$ Calibration $10 \mathrm{mV}, 1 \mathrm{~s}$. 

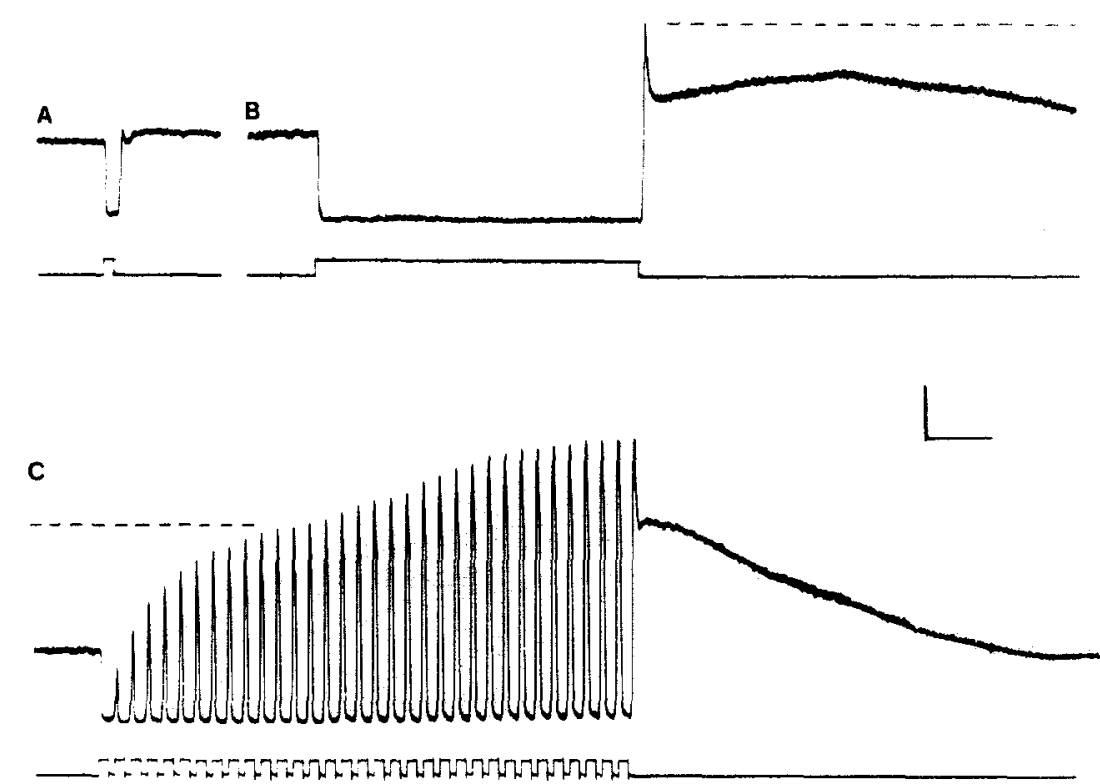

Fig. 4. Comparison of off-responses produced by steady and repetitive stimulation. A: response to single flash of $0.3 \mathrm{~s}$ duration. B: stimulus duration increased to $10 \mathrm{~s}$. C: repetitive stimulation with 0.3 -s flashes (same flashes as in A) separated by 0.2 -s dark intervals. Total exposure to light was the same as in $B$. Horizontal dashed line in $B$ and $C$ indicates maximum off-response amplitude that could be produced by increasing the duration of the stimulus. Calibration $10 \mathrm{mV}, 5 \mathrm{~s}$.

components of the off-response was reached with a stimulus duration of $15 \mathrm{~s}$. Since the dark potential and the amplitudes of the hyperpolarizing responses during illumination were the same in all of the responses, it is clear that the changes in the off-response did not depend on the amplitude of the preceding hyperpolarization during illumination, or on the level of the dark potential preceding the stimulus.

With increasing stimulus duration, there was also an increase in the rate of rise of the off-response, and a decrease in its latency. These changes can be seen in the records of Fig. 2, which shows, on a faster time scale, superimposed off-responses from another cell to flashes of different duration. Although not shown in these records, the membrane potential just before each light stimulus, and the time-course and latency of the hyperpolarizing response to the light stimuli (the on-responses) were the same in each case.

\section{Effects of repetitive stimulation on the off-response}

As shown above, the enhancement of the off-response appeared to be a condition which accumulated during exposure to light. It was not necessary that the light be continuous, however. A series of short flashes could also produce enhancement of the off-response.

Figure 3 shows, in another cell, the enhancement of both the fast and slow components of the off-response with repetitive stimulation. Trace $A$ shows the responses to 0.5 -s flashes separated by dark intervals of $3.5 \mathrm{~s}$. With this dark interval there was a slight enhancement of both the fast and slow components of the off-responses. Trace
B shows the responses to the same flashes when the dark interval was decreased to $1 \mathrm{~s}$. Both the fast and slow components of the off-response became larger after each successive flash, until they were several times larger than in the response to a single flash. The increased amplitude of the slow component caused a progressively greater depolarization in darkness between flashes, so that the amplitudes of the light-evoked hyperpolarization (onresponse) also increased in amplitude. In this experiment the light intensity was made bright enough to produce saturating responses in order to ensure that all of the off-responses arose from the same level of membrane

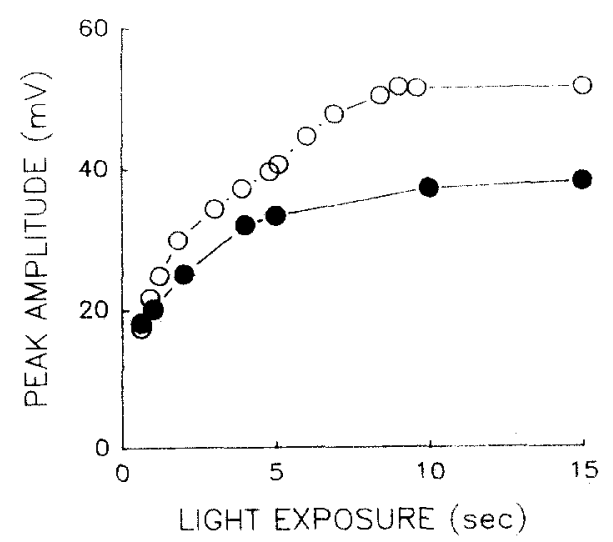

Fig. 5. Peak amplitude of off-response following steady (O)and repetitive $(O)$ stimulation. Ordinate, peak amplitude of fast component of off-response, measured as amount of depolarization. from the steady potential in light. Abscissa, total duration of light exposure (duration of individual steady stimuli, or the sum of durations of all flashes in a train of repetitive stimuli). 
potential. Therefore the enhancement of the off-response cannot be due to a change in the level of membrane potential during light.

In some respects the enhancement of off-responses produced by repetitive stimulation was different from that seen with increased stimulus duration. Repetitive stimulation often caused a greater enhancement of the off-response than the maximum that could be achieved by increasing the duration of the light stimulus. Fig. 4A shows the response of a cell to a brief $(0.3 \mathrm{~s})$ flash. With increasing stimulus duration the amplitude of both the fast and slow components of the off-response were increased. Fig. 4B shows the response to a 10-s flash, which was long enough to produce nearly maximum enhancement of both the fast and slow components of the off-response. Fig. 4C shows the responses of this cell to a train of repetitive stimulation with the same $0.3-\mathrm{s}$ flash as in A, separated by 0.2 -s dark intervals. The total amount of time in light in the train of flashes in $C$ was the same as in $B$, but the amplitude of the off-responses became much larger. In fact, the amount of enhancement produced by 10 -s steady illumination (indicated by a dashed line in $\mathrm{B}$ and $\mathrm{C}$ ) was reached after only $6 \mathrm{~s}$ of repetitive stimulation, during which time the actual exposure to light was only $3.6 \mathrm{~s}$.

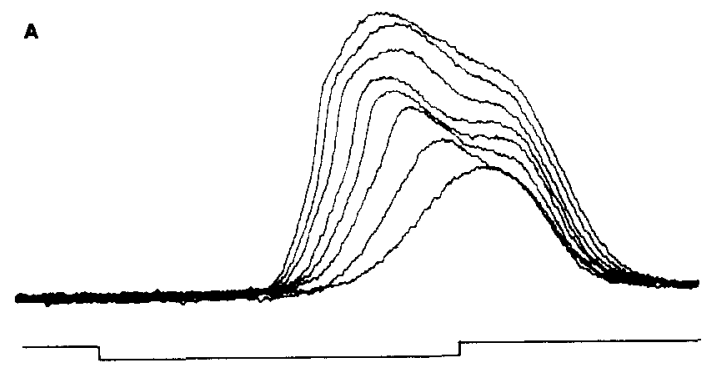

$\mathbf{B}$

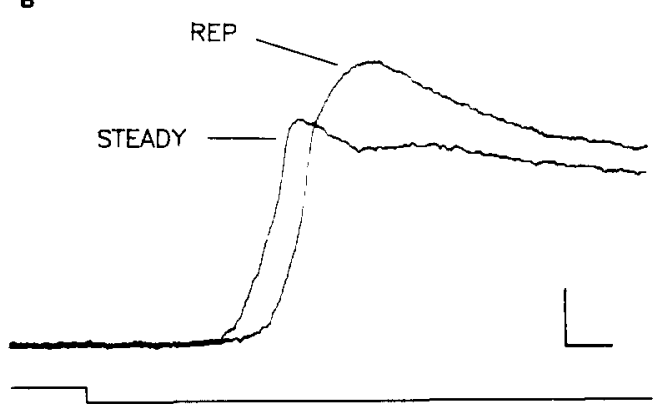

Fig. 6. Off-responses to steady and repetitive stimulation at faster time resolution. A: superimposed off-responses following flashes number $3,5,8,12,15,20,25$ and 30 in the train of repetitive stimuli shown in Fig. 4C. B: superimposed off-responses to $10 \mathrm{~s}$ duration steady light (STEADY), and to the last flash in the train of repetitive stimuli shown in Fig. 4C (REP). Total light exposure was $10 \mathrm{~s}$ in both cases. For all records, downward deflection of stimulus monitor indicates termination of light stimulus. Calibration $10 \mathrm{mV}$, $50 \mathrm{~ms}$.

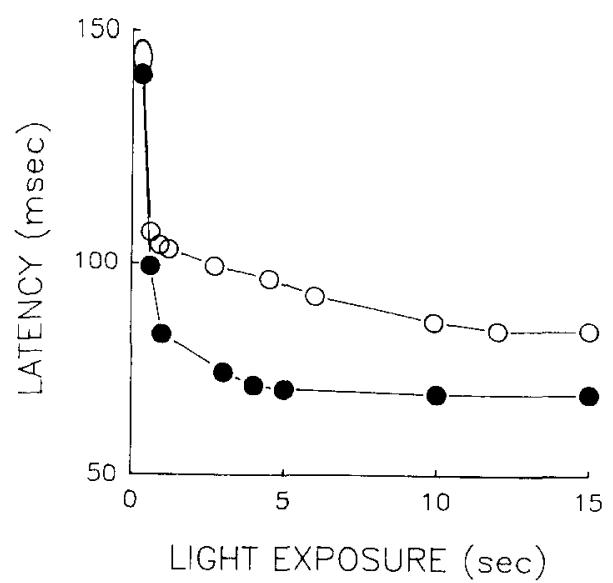

Fig. 7. Latency of off-responses following steady $(\boldsymbol{\theta})$ and repetitive (O) stimulation. Ordinate, latency of onset of off-response. Abscissa, total duration of light exposure, as described in Fig. 6.

Fig. 5 compares the amplitudes of off-responses produced by steady illumination (filled circles) and repetitive stimulation (open circles), for different total periods of exposure to light. In this graph the ordinate is the peak amplitude of the off-response. For repetitive stimuli, the off-response to the last flash in the series was measured. The abscissa is the total time of exposure to light. The two points for time $=10 \mathrm{~s}$ indicate the off-responses measured from the records in Figs. 4B and $4 \mathrm{C}$. Although with both types of stimulation the amplitude of the off-responses increased with increasing stimulus duration, for any given duration of illumination the amplitude was greater if the illumination was presented as a series of short flashes.

Fig. 6A shows, on a fast time scale, superimposed off-responses to different flashes during the repetitive stimulation of Fig. 4C. During repetitive stimulation with

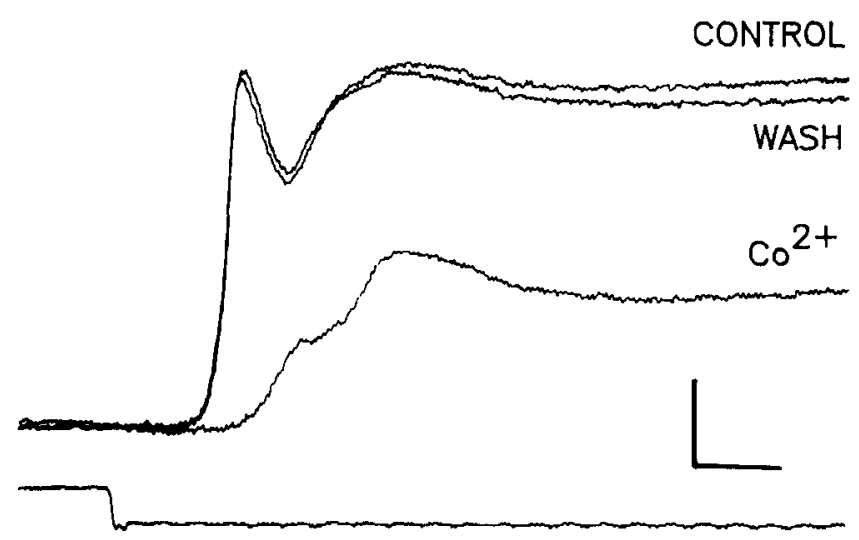

Fig. 8. Effect of cobalt on the off-response. Superimposed records of off-responses to flashes of $0.5 \mathrm{~s}$ duration. Onset of light response not shown; downward deflection of lower trace indicates end of light stimulus. Larger off-response was obtained in normal Ringer solution. Smaller response to same stimulus was recorded $5 \mathrm{~min}$ after switching to a solution containing $0.5 \mathrm{mM}$ cobalt chloride. Membrane potential during the hyperpolarizing light response was the same. Calibration bars $20 \mathrm{mV}, 50 \mathrm{~ms}$. 
a brief flash, successive off-responses showed a progressive increase in rate of rise and decrease in latency, much like those seen with increasing duration of the light stimulus in Fig. 2. However, the latencies of the off-responses following repetitive stimulation were significantly longer than those produced by the same duration of steady illumination. Fig. 6B shows superimposed off-responses produced by the single long stimulus in Fig. 4B and the last stimulus in the train of flashes in Fig. 4C. In both cases the total exposure to light was 10 s. Note that even though the off-response produced by repetitive stimulation was larger, it had a much longer latency. The graph of Fig. 7 shows similar comparisons for various durations of light exposure; for a given duration of light exposure the latency was always longer when the light was presented as a repetitive stimulation. This suggests that the mechanism of enhancement may be different in the two cases.

\section{Effect of decreasing calcium entry during the off-response}

It is not known whether the fast component of the off-response in turtle horizontal cells is generated in the horizontal cells themselves or in photoreceptors. Voltagedependent calcium currents have been described under certain conditions in fish and turtle horizontal cells ${ }^{9,11,15}$. To test whether the fast component of the off-response may involve a voltage-dependent calcium current, the effects of cobalt or low extracellular calcium were tested. Fig. 8 shows the effect of adding $0.5 \mathrm{mM}$ cobalt chloride to the bathing solution. The addition of cobalt caused a hyperpolarization of the dark potential and a reduction in amplitude of the off-response. However, it affected the fast component of the off-response to a much greater extent than the dark potential or the slow component, suggesting that calcium ions may be directly involved in the generation of this component, wherever its origin. Similar effects were seen when the bathing solution was switched to a nominally calcium-free solution. The effects of cobalt and low calcium were completely reversed when the test solutions were replaced with normal Ringer.

\section{DISCUSSION}

The off-responses of turtle horizontal cells at the termination of a light stimulus consist of several components which depend on both the intensity and duration of the preceding light stimuli. The present study deals only with the latter. The phenomena described here were observed in fresh preparations and after up to $4 \mathrm{~h}$ of recording, and thus do not appear to be associated with deterioration of the preparation. In most cells, increasing the duration of a light stimulus caused an increase in the rate of rise and a decrease in the latency of the initial depolarization, and an increase in the amplitudes of the fast and slow components. The increased amplitude of the slow component of the off-response resulted in an increase in amplitude of the hyperpolarizing response (on-response) to subsequent light stimuli which occurred during this period. The enhancement of the off-response appears to be a condition which develops or accumulates with increasing time in light and decays with increasing time in darkness.

Understanding the mechanism by which the offresponse is enhanced is complicated by the fact that it is not known whether it is generated in the horizontal cell itself or generated in photoreceptors and transmitted synaptically to horizontal cells. Unfortunately, it was not possible to determine this directly by recording from both cones and horizontal cells under the same conditions, since we were not able to record from cones in this preparation. Off-responses have been recorded from turtle cones ${ }^{12,14}$, but these responses were small compated to those seen in horizontal cells, and there is no indication that they showed the type of enhancement reported here. In any case, the absence of such effects in recordings from cone inner segments would not rule out their presence in cone terminals. For these reasons, we will assume in the following discusion that the offresponses recorded from horizontal cells and their enhancement are not solely events which are already present in the cone membrane potential, and that they could occur at cone terminals or in horizontal cells. Indeed, grounds for the latter assumption are that other adaptational changes in horizontal cell responses appear to be generated at sites distal to what is revealed in the cone membrane potential. For example, in horizontal cells of tiger salamander, light-induced changes in the waveform of the hyperpolarizing response to light onset (the on-response) have been attributed to postsynaptic actions $^{21.22}$. Also, horizontal cell responses may be affected by neuromodulator substances acting on transmission between photoreceptors and horizontal cells ${ }^{7,8,19}$. Normann and Perlman ${ }^{12}$ discussed in some detail how changes in the gain of synaptic transmission between photoreceptors and horizontal cells might be due to either an increase in release of transmitter or an increase in sensitivity to transmitter. These two possibilities are considered below in relation to the present results.

First, the process of transmitter release may somehow be altered as a result of the preceding illumination, in a way that is not reflected in the membrane potential of the photoreceptor. For example, there could be some desensitization in the transmitter release process during steady darkness, with a time-dependent recovery during subsequent illumination.

Second, the time-course of the off-response in hori- 
zontal cells may be altered by a change in their sensitivity to the transmitter released by photoreceptors. The glutamate receptors on horizontal cells may be partially desensitized by the relatively high level of glutamate present in darkness, and this desensitization may be progressively removed with increasing time in light. There is conflicting evidence as to whether there is desensitization of isolated horizontal cells to glutamate ${ }^{4-6,9.13}$, but very rapid desensitization of glutamate receptors has been observed in other neurons $\mathrm{s}^{3,16}$.

It is also possible that the enhancement of the off-responses is in part due to some membrane property of horizontal cells, for example the slow removal of inactivation of a voltage-dependent inward current during the light-evoked hyperpolarization. However, this possibility could not be tested directly by altering the membrane potential with extrinsic current because of the low input resistance of the horizontal cell network.

Repetitive stimulation caused changes similar to those produced by increasing the duration of the light stimulus, but in some cases it also produced an additional enhancement which could not be obtained by increasing the duration of the stimulus. Part of the effect of repetitive stimulation may be the same as with increased stimulus duration, i.e. the cumulative effect of the light stimuli, diminished by the partial loss of this enhancement during the dark intervals. But with repetitive stimulation the amount of enhancement of the off-response was greater, and the latency was longer than could be obtained by increasing the stimulus duration. Thus the enhancement of the off-responses appears to involve two separate mechanisms, one related to the cumulative effect of illumination and another which seems to be the result of the repetitive nature of the stimulus. Effects of repetitive stimulation which might contribute to the additional enhancement might be the repeated depolarizations of the horizontal cells, which could release more feedback

\section{REFERENCES}

1 Cervetto, L. and MacNichol, E.F. Inactivation of horizontal cells in turtle retina by glutamate and aspartate, Science, 178 (1972) 767-768.

2 Copenhagen, D.R. and Jahr, C.E., Release of excitatory amino acids from turtle photoreceptors, Nature, 341 (1989) 536-539.

3 Dudel, J., Franke, C., Hatt, H., Ramsey, R.L. and Usherwood, P.N.R., Rapid activation and desensitization by glutamate of excitatory, cation-selective channels in locust muscle, Neurosci. Lett., 88 (1988) 33-38.

4 Hals, G., Christensen, B.N., O'Dell, T., Christensen, M. and Shingai, R., Voltage-clamp analysis of currents produced by glutamate and some glutamate analogues on horizontal cells isolated from the catfish retina, $J$. Neurophysiol., 56 (1986) 19-31.

5 Ishida, A.T., Kaneko, A. and Tachibana, M., Responses of solitary retinal horizontal cells from Carassius auratus to 1.glutamate and related amino acids, J. Physiol. (Lond.), 348 transmitter, or increased release of a modulator substance by some other neuron.

\section{It seems unlikely that the enhancement of the off-} responses during repetitive stimulation was due to the increased depolarization following the previous flash, as was proposed for tiger salamander horizontal cells ${ }^{20}$, since the second off-response in the series in Fig. $4 \mathrm{C}$ was larger than the first, even though the dark potential at the onset of the second flash was more negative than at the onset of the first flash. Figure $4 \mathrm{C}$ also shows that enhancement occurs even when the dark interval between flashes is so short that the slow component does not appear.

The light-dependent enhancement of the off-responses in horizontal cells is a form of light adaptation, which allows the retina to respond optimally under changing conditions of illumination. It enhances the response to dimming, as well as the on-responses to rapidly changing stimuli. The complex responses sometimes seen in other retinal neurons at the termination of a light stimulus may be partly due to the phenomena described here in horizontal cells. It is likely that at least some of this enhancement mechanism involves changes in the process of synaptic transmission between photoreceptors and horizontal cells. Other examples of modulation of synaptic transmission at this level in the retina include the effects of neuromodulator substances such as dopamine ${ }^{7}$, ${ }^{8,19}$ and possibly $\mathrm{GABA}^{18,22}$ and glycine ${ }^{18}$ on transmission between photoreceptors and horizontal cells, as well as light-dependent morphological changes in the synaptic connections between horizontal cells and cones ${ }^{17}$. This short-term potentiation of horizontal cell responses is thus another example of neuronal plasticity in the outer plexiform layer of the retina.

Acknowledgements. Supported by the Deutsche Forschungsgemeinschaft and NIH Grant EY-01653. We thank Dr. Josef Ammermüller for reading the manuscript.

(1983) 255-270.

6 Ishida, A. and Neyton, J., Quisqualate and L-glutamate inhibit retinal horizontal-cell responses to kainate, Proc. Natl. Acad. Sci. U.S.A., 82 (1985) 1837-1841.

7 Knapp, A.G. and Dowling, J.E., Dopamine enhances excitatory amino acid-gated conductances in cultured retinal horizontal cells, Nature, 325 (1987) 437-439.

8 Knapp, A.G., Schmidt, K.F. and Dowling, J.E., Dopamine modulates the kinetics of ion channels gated by excitatory amino acids in retinal horizontal cells, Proc. Natl. Acad. Sci. U.S.A., 87 (1990) 767-771.

9 Lasater, E.M. and Dowling, J.E., Carp horizontal cells in culture respond selectively to L-glutamate and its agonists, Proc. Natl. Acad. Sci. U.S.A., 79 (1982) 936-940.

10 Murakami, M., Ohtsu, K. and Ohtsuka, T., Effects of chemicals on receptors and horizontal cells in the retina, J. Physiol. (Lond.), 227 (1972) 899-913.

11 Murakami, M. and Takahashi, K.-I., Calcium action potential and its use for measurement of reversal potentials of horizontal 
cell responses in carp retina, J. Physiol. (Lond.), 386 (1987) 165-180.

12 Normann, R.A. and Perlman, I., Signal transmission from red cones to horizontal cells in the turtle retina, J. Physiol. (Lond.), 286 (1979) 509-524.

13 Perlman, I., Knapp, A.G. and Dowling, J.E., Responses of isolated white perch horizontal cells to changes in the concentration of photoreceptor transmitter agents, Brain Research, 487 (1989) 16-25.

14 Schwartz, E.A., Responses of bipolar cells in the retina of the turtle, J. Physiol. (Lond.), 236 (1974) 211-224.

15 Tachibana, M., Membrane properties of solitary horizontal cells isolated from goldfish retina, J. Physiol. (Lond.), 321 (1981) 141-161.

16 Trussell, L.O., Thio, L.L., Zorumski, C.F. and Fischbach, G.D., Rapid desensitization of glutamate receptors in vertebrate central neurons, Proc. Natl. Acad. Sci. U.S.A., 85 (1988) $4562-4566$.
17 Weiler, R. and Wagner, H.-J. i.ight-dependent change of cone-horizontal cell interactions in carp retina, Brain Research, 298 (1984) $1-9$.

18 Witkovsky, P. and Stone, S., GABA and glycine modify the balance of rod and cone inputs to horizontal cells in the Xenopus retina, Exp. Biol., 47 (1987) 13-22

19 Witkovsky, P., Stone, S. and Besharse, J.C.. Dopamine modifies the balance of rod and cone inputs to horizontal cells of the Xenopus retina, Brain Research, 449 (1988) 332-336.

$20 \mathrm{Wu}$, S.M., The off-overshoot of photoreceptors and horizontal cells in the light-adapted retinas of the tiger salamander, Exp. Eye Res., 47 (1988) 261-268.

21 Yang, X.-L. and Wu, S.M., Effects of background illumination on horizontal cell responses in the tiger salamander retina, $J$. Neurosci., 9 (1989) 815-826.

22 Yang, X.-L. and Wu, S.M., Effects of prolonged light exposure, GABA, and glycine on horizontal cell responses in tiger salamander retina, J. Neurophysiol., 61 (1989) 1025-1035. 